\title{
Interactive comment on "Climate Assessment Platform of Different Aircraft Routing Strategies in the Chemistry-Climate Model EMAC 2.41: AirTraf 1.0" by Hiroshi Yamashita et al.
}

Hiroshi Yamashita et al.

hiroshi.yamashita@dlr.de

Received and published: 17 May 2016

We are most grateful to the referee \#1 for the very helpful and encouraging comments on the original version of our manuscript. Please find our replies uploaded as the Supplement file (PDF).

Please also note the supplement to this comment: http://www.geosci-model-dev-discuss.net/gmd-2015-272/gmd-2015-272-AC1supplement.pdf 